\title{
Liver kinase B1/adenosine monophosphate-activated protein kinase signaling axis induces p21/WAF1 expression in a p53-dependent manner
}

\author{
QING MA $^{1}$, PING XIAO ${ }^{1}$, LINLIN SUN $^{2}$, JING WANG $^{2}$ and DIANSHENG ZHONG ${ }^{1,2}$ \\ ${ }^{1}$ Department of Medical Oncology, Tianjin Medical University General Hospital; ${ }^{2}$ Tianjin Key Laboratory of \\ Lung Cancer Metastasis and Tumor Microenvironment, Lung Cancer Institute, Tianjin Medical \\ University General Hospital, Tianjin, Hebei 300052, P.R. China
}

Received December 25, 2015; Accepted May 23, 2017

DOI: $10.3892 / \mathrm{ol} .2018 .8741$

\begin{abstract}
Liver kinase B1 (LKB1) encodes a serine/threonine kinase and functions as a tumor suppressor. LKB1 loss-of-function somatic mutations are frequently observed in sporadic types of cancer, particularly in lung cancer. Ectopic LKB1 induces growth arrest by upregulating p21/cyclin dependent kinase inhibitor 1A (WAF1) in LKB1 deficient cervical and melanoma cancer cell lines. However, the underlying molecular mechanism remains to be elucidated. The present study built upon previous observations by confirming that the ectopic expression level of LKB1 significantly reduced colony formation of LKB1-deficient lung cancer cells. Mechanistically, the present study demonstrated that LKB1 overexpression significantly induced p21/WAF1 expression in a kinase-dependent manner. Conversely, LKB1 stable knockdown resulted in a decrease in p21/WAF1 expression level in colon cancer cells. In addition, it was revealed that pharmacological activation of adenosine monophosphate protein kinase (AMPK) by 2-deoxyglucose significantly increased the p21/WAF1 expression level, suggesting that AMPK acts downstream of LKB1 to induce p21/WAF1 expression. Furthermore, the present study demonstrated that functional p53 was required for $\mathrm{p} 21 / \mathrm{WAF} 1$ induction by LKB1. Phosphorylation of p53-Ser ${ }^{15}$ was increased by ectopic LKB1 or AMPK activation. Taken together, these results suggested that LKB1 acts via its substrate, AMPK, to upregulate p21/WAF1 expression in a p53-dependent manner. Therefore, the present study identified an important signaling axis, providing novel molecular insights into the tumor suppressor role of LKB1.
\end{abstract}

Correspondence to: Dr Diansheng Zhong, Department of Medical Oncology, Tianjin Medical University General Hospital, 154 Anshan Road, Heping, Tianjin, Hebei 300052, P.R. China

E-mail: zhongdsh@hotmail.com

Key words: liver kinase B1, p21/cyclin dependent kinase inhibitor $1 \mathrm{~A}$, adenosine monophosphate protein kinase, p53

\section{Introduction}

Liver kinase B1 (LKB1), also known as serine/threonine kinase 11 , is a well-established tumor suppressor. Germline mutations in LKB1 are associated with Peutz-Jeghers syndrome, a disorder characterized by benign hamartomas of the gastrointestinal tract (1) and is predisposed to certain types of cancer (2). LKB1 somatic mutations are frequently identified in sporadic types of cancer, particularly in lung cancer $(20-30 \%)(3,4)$, ranking it as the third most frequently mutated gene in lung adenocarcinoma following p53 and K-Ras (5). As a serine/threonine protein kinase, LKB1 acts as a master upstream kinase of a group of adenosine monophosphate-activated protein kinases (AMPKs), and is involved in a wide range of cellular functions $(6,7)$.

AMPK, one of the major substrates of LKB1, is an energy sensor and metabolic switch. AMPK is activated by LKB1 under energy stresses, including adenosine triphosphate (ATP) depletion induced by glycolysis inhibitors. 2-deoxyglucose (2-DG) is a well-characterized glycolysis inhibitor (8). 2-DG is converted by hexokinase to 2-DG-P, which cannot be further metabolized, thus is trapped inside the cell and inhibits hexokinase. The inhibition of glycolysis by 2-DG treatment induces a decrease in intracellular ATP concentration and an increase in intracellular AMP concentration. The increased AMP expression level binds to AMPK and alters its conformation, resulting in AMPK activation by LKB1 via phosphorylation of AMPK at Thr172 (9).

As a tumor suppressor, LKB1 was previously demonstrated to arrest cell cycle and inhibit cancer cell growth by inducing p21/cyclin dependent kinase inhibitor 1A (WAF1) (10-13). However, the molecular mechanism underlying p21/WAF1 induction by LKB1 is not well understood. Firstly, it remains an open question whether LKB1-mediated p21/WAF1 induction is specific to certain cancer cell lines or whether it is a general characteristic. In addition, the substrate molecule downstream of LKB1 mediating p21/WAF1 induction has not yet been identified. Finally, there have been contradicting findings on whether functional p53, the key transcription factor of p21/WAF1 (14), is required for p21/WAF1 induction by LKB1. The present study built upon previous studies (10-12) by 
confirming that ectopic LKB1-induced p21/WAF1 expression occurs in lung and colon cancer cells. Mechanistically, it was revealed that $\mathrm{p} 53$ was required for $\mathrm{p} 21 / \mathrm{WAF} 1$ upregulation by LKB1. In addition, the results of the present study suggested that AMPK may act downstream of LKB1 to increase p21/WAF1 expression, possibly by phosphorylating $\mathrm{p} 53-\mathrm{Ser}^{15}$. Taken together, the results demonstrated that LKB1 acts via its substrate AMPK to induce p21/WAF1 expression in a p53-dependent manner. Therefore, the results of the present study have shed new light on the molecular mechanism underlying the tumor suppressor role of LKB1.

\section{Materials and methods}

Materials. Mouse monoclonal antibody against LKB1 was purchased from Abcam (Cambridge, UK). Antibodies against p21/WAF1, phosphorylated (p)-p53-Ser ${ }^{15}$ and p-AMPK-Thr 172 were purchased from Cell Signaling Technology, Inc. (Danvers, MA, USA). Mouse anti-GAPDH antibody was purchased from Santa Cruz Biotechonology, Inc. (Dallas, TX, USA). 2-DG was purchased from Sigma-Aldrich (Merck KGaA, Darmstadt, Germany). The pEGFP-C2, pEGFP-LKB1 and pEGFP-LKB1-K78M plasmids were provided by Professor Wei Zhou (The Winship Cancer Institute, Emory University School of Medicine, Atlanta, GA, USA). The lentiviral LKB1 short hairpin RNA (shRNA) construct and a negative control construct, which was created in the same vector system (pLKO.1), were purchased from Thermo Fisher Scientific, Inc. (Waltham, MA, USA). Lentiviral helper plasmids, pCMV-dR8.2 dvpr and pCMV-VSV-G, were obtained from Addgene, Inc. (Cambridge, MA, USA).

Cell lines and cell culture. A549 and H460 lung cancer cell lines were purchased from the American Type Culture Collection (Manassas, VA, USA). The p53 wild-type HCT116 and the isogenic HCT116 $\mathrm{p}^{-/} 3^{-}$colon cell lines were provided by Professor Wei Zhou (The Winship Cancer Institute, Emory University School of Medicine). Cells were cultured in RPMI-1640 medium supplemented with 10\% fetal bovine serum (both from Gibco; Thermo Fisher Scientific Inc.) at $37^{\circ} \mathrm{C}$ in a humidified atmosphere with $5 \% \mathrm{CO}_{2}$.

Western blotting. Following treatment, cells were lysed on ice for 20 min using the radioimmunoprecipitation assay lysis and extraction buffer (cat. no. 89900; Thermo Fisher Scientific, Inc.). The protein concentration of each cell sample was determined using the bicinchoninic acid protein assay reagent (Thermo Fisher Scientific, Inc.). A total of $20 \mu \mathrm{g}$ protein/lane was denatured in sodium dodecyl sulfate sample buffer (cat. no. 9173; Takara Biotechnology Co., Ltd., Dalian, China) and separated using SDS-PAGE (10\% gel). Subsequently, proteins were transferred onto polyvinylidene difluoride membranes (EMD Millipore, Billerica, MA, USA). Followed blocking with $5 \%$ skimmed milk for $1.5 \mathrm{~h}$ at room temperature, the membranes were incubated with the recommended dilutions of primary antibodies against LKB1 (cat. no. ab-15095; dilution, 1:1,000; Santa Cruz Biotechnology, Inc.), p21/WAF1 (cat. no. 2947; dilution, 1:1,000; Cell Signaling Technology, Inc.), p-AMPK (cat. no. 8208; dilution, 1:1,000; Cell Signaling Technology, Inc.), p-p53 Ser ${ }^{15}$ (cat. no. 9284; dilution,
1:1,000; Cell Signaling Technology, Inc.), p53 (cat. no. 9282; dilution, 1:1,000; Cell Signaling Technology, Inc.) and GAPDH (cat. no. sc-47724; dilution, 1:3,000; Santa Cruz Biotechnology, Inc.) at $4^{\circ} \mathrm{C}$ overnight and rinsed twice with PBS. The membranes were subsequently incubated with the following secondary antibodies: Horseradish peroxidase (HRP)-conjugated anti-rabbit immunoglobulin G (IgG; cat. no. ZB2301; dilution, 1:10,000; OriGene Technologies, Inc.) or the HRP-conjugated anti-mouse $\operatorname{IgG}$ antibody (cat. no. ZB2305; dilution, 1:10,000; OriGene Technologies, Inc.) at room temperature for $2 \mathrm{~h}$. Peroxidase-labeled bands were visualized using an enhanced chemiluminescence detection reagent (Pierce; Thermo Fisher Scientific, Inc.), according to the manufacturer's protocol. Results were analyzed using Image software (version 1.5; National Institutes of Health, Bethesda, MD, USA) to compare the relative target protein expression.

Colony formation assay. A549 cells were plated at a density of $2 \times 10^{5}$ cells/well in six-well plates overnight at $37^{\circ} \mathrm{C}$ in a humidified incubator containing $5 \% \mathrm{CO}_{2}$. The following day, cells were transfected with plasmids encoding wild-type LKB1 or mutant LKB1-K78M (kinase-deficient) in duplicate with Lipofectamine ${ }^{\circledR} 2000$ reagent (Invitrogen; Thermo Fisher Scientific, Inc.). A total of $2 \mu \mathrm{g}$ plasmid and $6 \mu \mathrm{l}$ Lipofectamine 2000 were added to each well. Cells were selected using G418 $(2 \mathrm{mg} / \mathrm{ml}) 72 \mathrm{~h}$ after transfection for 2 weeks at $37^{\circ} \mathrm{C}$ in a humidified incubator containing $5 \% \mathrm{CO}_{2}$. The medium was replaced every 4 days. Finally, the cells were fixed with $10 \%$ trichloroacetic acid solution for $20 \mathrm{~min}$ and stained with $0.5 \%$ crystal violet for $30 \mathrm{~min}$ at room temperature. The stained cells were washed and air-dried, then observed under a light microscope (x40, magnification). Colony numbers were assessed visually and colonies containing $>50$ normal-appearing cells were counted. Statistical differences in colony numbers between the wild-type LKB1 and LKB1-K78M plasmid-transfected cells were evaluated using the two-tailed Student's t-test.

LKB1 stable knockdown using lentiviral shRNA. Lentivirus stocks were prepared according to the manufacturer's protocol, as previously described (15). Briefly, $1.5 \times 10^{6} 293 \mathrm{~T}$ cells were plated in $10-\mathrm{cm}$ dishes. Cells were co-transfected with shRNA constructs $(3 \mu \mathrm{g})$ together with pCMV-dR8.2 dvpr $(3 \mu \mathrm{g})$ and pCMV-VSV-G $(0.3 \mu \mathrm{g})$ helper constructs. Following 2 days of incubation at $37^{\circ} \mathrm{C}$ in a humidified atmosphere containing $5 \%$ $\mathrm{CO}_{2}$, viral stocks were harvested from the culture medium, which was filtered to remove non-adherent $293 \mathrm{~T}$ cells. In order to select the colon cancer cells that were stably expressing shRNA constructs, HCT116 cells were plated at sub-confluent densities, and infected with a cocktail of $1 \mathrm{ml}$ virus-containing RPMI medium, $3 \mathrm{ml}$ RPMI-medium (no antibiotics, heat inactivated serum) and $8 \mu \mathrm{g} / \mathrm{ml}$ polybrene at $37^{\circ} \mathrm{C}$ in a humidified atmosphere with $5 \% \mathrm{CO}_{2}$. Selection with $2 \mu \mathrm{g} / \mathrm{ml}$ puromycin was initiated $48 \mathrm{~h}$ after lentivirus infection. Following $\sim 4$ weeks of selection, monolayers of stably infected pooled clones were harvested for use and cryopreserved.

$R N A$ extraction and quantitative polymerase chain reaction (qPCR). Total RNA was isolated from HCT116-pLKO.1 and HCT116-LKB1 isogenic colon cancer cell lines using TRIzol ${ }^{\circledR}$ 
reagent (Invitrogen; Thermo Fisher Scientific, Inc.). The first-strand cDNA was prepared using the PrimeScript First strand cDNA Synthesis kit (Takara Bio, Inc.), according to the manufacturer's protocol, using $1 \mu \mathrm{g}$ total RNA. All qPCR reactions were performed in a $20 \mu \mathrm{l}$ mixture containing $1 \mathrm{X}$ SYBR Green supermix (Takara Bio, Inc.), $0.2 \mu \mathrm{mol} / 1$ of each primer and $2 \mu \mathrm{l}$ cDNA template. Primers for LKB1 were as follows: Forward, 5'-CTGGGGTCACCCTCTACAAC-3'; and reverse, 5'-ACTCAAGCATCCCTTTCAGC-3'. Primers for GAPDH were as follows: Forward, 5'-GGAGTCAACGGA TTTGGTCG-3'; and reverse, 5'-CTTGATTTTGGAGGGATC TCG-3'. qPCR was performed using the Applied Biosystems 7500 system (Applied Biosystems; Thermo Fisher Scientific, Inc.) under the following cycling conditions: (Step 1) $95^{\circ} \mathrm{C}$ for $30 \mathrm{sec}$; (step 2) 40 cycles of $95^{\circ} \mathrm{C}$ for $5 \mathrm{sec} ; 60^{\circ} \mathrm{C}$ for $34 \mathrm{sec}$; the melting curve stage was followed. The relative expression level of LKB1 was normalized to GAPDH. Relative mRNA concentrations were determined using the $2^{-\Delta \Delta \mathrm{Cq}}$ method (16).

RNA interference. The LKB1 small interfering RNA (siRNA) sequence was as follows: Sense, 5'-CCAACGUGAAGAAGG AAAUTT-3' and antisense, 5'-AUUUCCUUCACGUUG GTT-3'. The control siRNA sequences was as follows: Sense, 5'-UUCUCCGAACGUGUCACGUTT-3' and antisense, 5'-ACG UGACACGUUCGGAGAATT-3'. All siRNAs were synthesized by Shanghai Genechem Co., Ltd. (Shanghai, China). The HCT116 and HCT116p53-/- cancer cell lines were used for RNA interference. Cells were plated at a density of $2 \times 10^{5}$ cells/well in 6-well plates overnight at $37^{\circ} \mathrm{C}$ in a humidified incubator containing $5 \% \mathrm{CO}_{2}$. Cells were subsequently transfected with $100 \mathrm{nmol} / 1$ of LKB1 siRNA or negative control siRNA using the Lipofectamine ${ }^{\circledR} 2000$ reagent (Invitrogen; Thermo Fisher Scientific Inc.), according to the manufacturer's protocol. For every 105 cells, $0.5 \mu \mathrm{g}$ LKB1 siRNA and control siRNA were diluted and mixed with $3 \mu \mathrm{l}$ transfection reagent. After incubation for $30 \mathrm{~min}$ at room temperature, the transfection mixture was added to the cells. Following $6 \mathrm{~h}$ of incubation at $37^{\circ} \mathrm{C}$ in a humidified incubator containing $5 \% \mathrm{CO}_{2}$, the RPMI-1640 medium was replaced with serum-enriched 1640-RPMI medium (RPMI-1640 medium supplemented with 10\% fetal bovine serum) and the cells were cultured for an additional $48 \mathrm{~h}$ at $37^{\circ} \mathrm{C}$ in a humidified incubator containing $5 \% \mathrm{CO}_{2}$. Subsequently, the transfected cells were collected.

Statistical analysis. SPSS software (version 17; IBM Corporation, NY, USA) was used for statistical analysis of the results. The majority of the results are representative $\geq 3$ independent experiments and are presented as the mean \pm standard deviation of triplicate samples. Error bars represent standard deviations between experiments. One-way analysis of variance and Student's t-test ( $\leq 2$ groups) were used to determine statistical significance. Fisher's least significant difference post-hoc test was used to compare the differences between $\geq 3$ groups. $\mathrm{P}<0.05$ was considered to indicate a statistically significant difference.

\section{Results}

Restoring LKB1 activity in lung cancer cells induces growth suppression. To investigate whether the kinase activity of LKB1 is required for growth inhibition in lung cancer cells, LKB1 mutant A549 cells were transfected with plasmids encoding wild-type LKB1 or mutant LKB1-K78M (kinase-deficient). Subsequently, these transfections were subjected to G418 $(2 \mathrm{mg} / \mathrm{ml})$ selection for 14 days. As presented in Fig. 1A and B, ectopic expression of wild-type LKB1 resulted in a significantly reduced number of colonies compared with those transfected with LKB1-K78M. In order to evaluate the transfection efficiency of LKB1, the protein expression level of LKB1 was evaluated using western blotting. As presented in Fig. 1C, the expression level of wild-type LKB1 and the mutant were similar, suggesting equal transfection efficiencies. This result suggests that the kinase activity of LKB1 is required for the suppression of lung cancer cell proliferation.

LKB1 upregulates p21/WAF1 expression in lung and colon cancer cells. p21/WAF1 serves an essential role in arresting cell cycle and inducing growth suppression; therefore, the present study suggested that p21/WAF1 may be functionally important for the tumor suppressive effects of LKB1 in lung cancer cells. To evaluate whether LKB1 regulates p21/WAF1 expression in NSCLC cells, LKB1 mutant A549 and H460 cells were used. According to our previous study, H460 and A549 cells harbor a nonsense mutation at codon 37 of LKB1, thus LKB1 protein is not expressed in these cells lines (15). The cells were transfected with plasmids encoding wild-type LKB1, LKB1 K78M or vector, and the protein expression level of p21/WAF1 was determined through western blotting. As presented in Fig. 2A, compared with the control group, ectopic LKB1 significantly increased p21/WAF1 protein expression level in both cell lines. Conversely, kinase deficient LKB1-K78M did not have a significant effect on p21/WAF1 expression level. These results suggest that the kinase activity of LKB1 is required to upregulate p21/WAF1 expression in lung cancer cells.

In order to validate this observation in other types of cancer cells, the LKB1 wild-type colon cancer cell line HCT116 was used. Using the lentivirus system, isogenic HCT116-pLKO.1 and HCT 116-LKB1 shRNA cell lines were established. As presented in Fig. 2B and C, compared with the control group, LKB1 mRNA and protein expression levels were significantly suppressed in HCT 116 cells stably expressing LKB1 shRNA. Additionally, it was revealed that LKB1 knockdown significantly decreased the expression level of p21/WAF1 (Fig. 2C), confirming that LKB1 positively regulated p21/WAF1 expression level in colon cancer cells.

AMPK acts downstream of LKB1 to regulate p21/WAF1 expression level. It has been reported that deficiency of AMPK- $\alpha 1$, a canonical substrate of LKB1 as described above, reduces p21/WAF1 expression level in mouse embryonic fibroblasts (17). Thus, the present study suggested that LKB1 acts indirectly to induce $\mathrm{p} 21 / \mathrm{WAF} 1$ expression via its substrate AMPK. To investigate this hypothesis, 2-DG, a glycolysis inhibitor and AMPK activator, was used. HCT116 cells were treated with $25 \mathrm{mM}$ 2-DG for $24 \mathrm{~h}$, the expression levels of p-AMPK Thr172 and p21/WAF1 were evaluated using western blotting. As presented in Fig. 3A, 2-DG treatment induced AMPK phosphorylation and significantly enhanced p21/WAF1 expression, suggesting that AMPK activation serves a role in p21/WAF1 induction. 
A

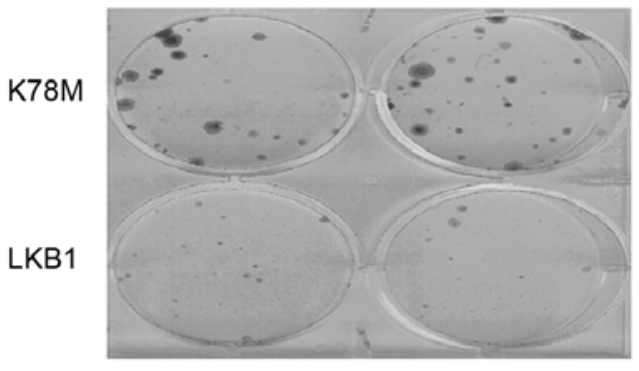

B

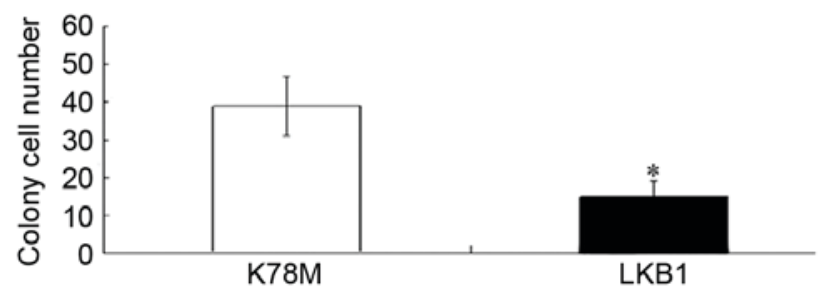

C

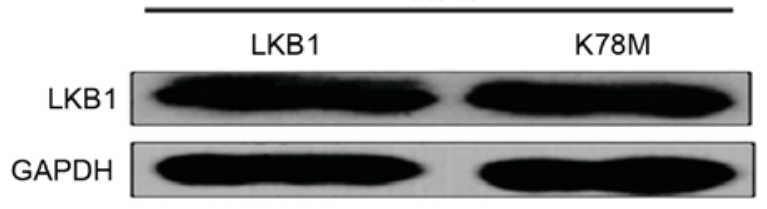

Figure 1. Restoring LKB1 activity in lung cancer cells induces growth suppression. (A) A549 cells were transfected with plasmids encoding wild-type LKB1 or mutant LKB1-K78M (kinase-deficient) in duplicate, selected with G418 $(2 \mathrm{mg} / \mathrm{ml}) 72 \mathrm{~h}$ after transfection for 2 weeks and subsequently colony formation assays were performed. Colony numbers were assessed visually and colonies containing $>50$ normal-appearing cells were counted. (B) Experiments were performed as in (A), and colony numbers were evaluated using the two-tailed Student's t-test. Results are presented as the mean \pm standard error of the mean from three independent experiments. ${ }^{\mathrm{P}}<0.05$ vs. the control. (C) Experiments were performed as in (A) and LKB1 protein expression level was determined through western blotting with GAPDH as the loading control. Results are presented as the mean \pm standard deviation. LKB1, liver kinase B1.

2-DG also demonstrated an off-target effect, which is independent of LKB1/AMPK signaling. To confirm that 2-DG induced p21/WAF1 expression mediated by LKB1/AMPK signaling, the isogenic HCT116-pLKO.1 and HCT116-LKB1 shRNA cell lines were treated with $25 \mathrm{mM}$ 2-DG for $24 \mathrm{~h}$. As presented in Fig. 3B, 2-DG activated AMPK and significantly increased p21/WAF1 expression in HCT116-pLKO.1 cells. In contrast, in HCT116-LKB1 shRNA cells, 2-DG failed to induce AMPK activation, and LKB1 depletion diminished the 2-DG-inducing effects on p21/WAF1. In addition, it was revealed that the phosphorylation of $\mathrm{p} 53-\mathrm{Ser}^{15}$ was increased following treatment with 2-DG, which was significantly attenuated by LKB1 loss. These results suggest that AMPK acts downstream of LKB1 to induce p21/WAF1 expression.

LKB1/AMPK requires $p 53$ to regulate $p 21 / W A F 1$. The phosphorylation of $\mathrm{p} 53 \mathrm{Ser}^{15}$ is important for the stabilization of p53 protein (18), thus the present study suggested that p53 may be involved in p21/WAF1 induction by LKB1/AMPK. To determine whether p53 is required for LKB1-mediated p21/WAF1 induction, the p53 wide-type HCT116 colon cell
A

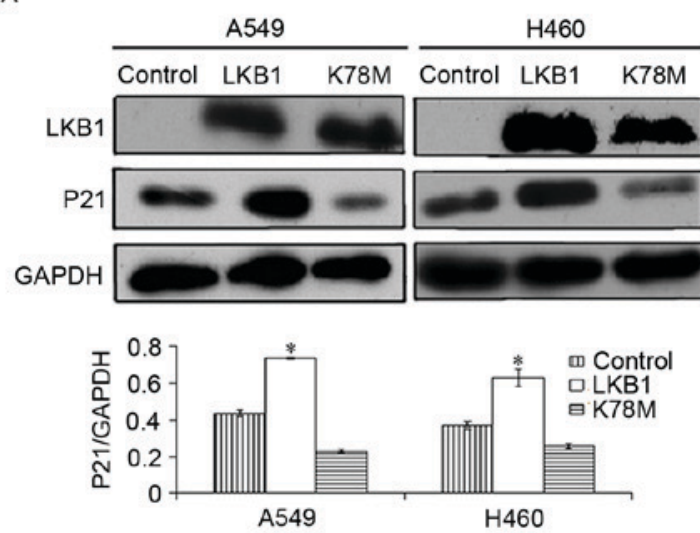

B

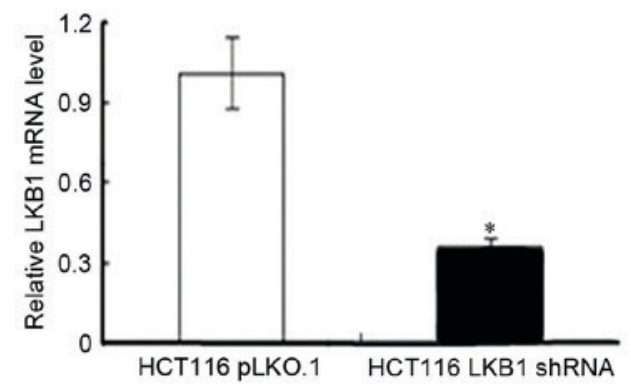

c
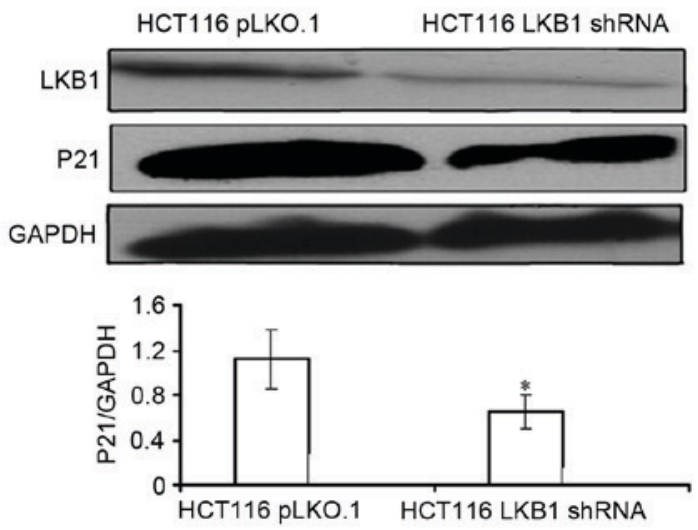

Figure 2. LKB1 upregulates p21/WAF1 expression level in lung and colon cancer cells. (A) LKB1 mutant A549 and H460 NSCLC cells were transfected with plasmids encoding wild-type LKB1, LKB1 K78M or vector, and LKB1 and p21/WAF1 protein expression level was examined using western blotting with GAPDH used as the loading control. (B) Isogenic colon cancer cell lines, HCT116-pLKO.1 and HCT116-LKB1 shRNA, were established using the lentivirus system. LKB1 mRNA expression level was determined using quantitative polymerase chain reaction normalized to GAPDH. (C) The cell lysates of isogenic HCT116-pLKO.1 and HCT116-LKB1 shRNA cells were collected, and the LKB1 and p21/WAF1 expression level was analyzed using western blotting. GAPDH served as a loading control. ${ }^{*} \mathrm{P}<0.05$ vs. the control. Each blot is representative of three blots obtained from three independent experiments. Results are presented as the mean \pm standard deviation. NSCLC, non-small cell lung cancer; LKB1, liver kinase B1; shRNA, short hairpin RNA; WAF1, cyclin dependent kinase inhibitor $1 \mathrm{~A}$.

line and its isogenic clones with $\mathrm{p} 53$ depletion (HCT116 $\mathrm{p} 53^{-1-}$ ) were used. As presented in Fig. 4A, the protein expression level of p53 was almost undetectable in HCT116 p53 ${ }^{-/}$cells. These cells were then transiently transfected with control short interfering (si)RNA or LKB1 siRNA, and the expression 
A

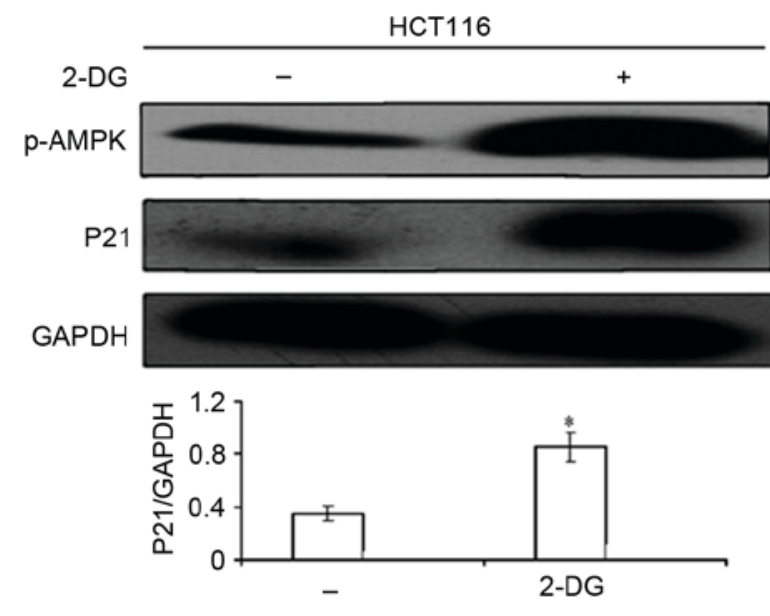

B

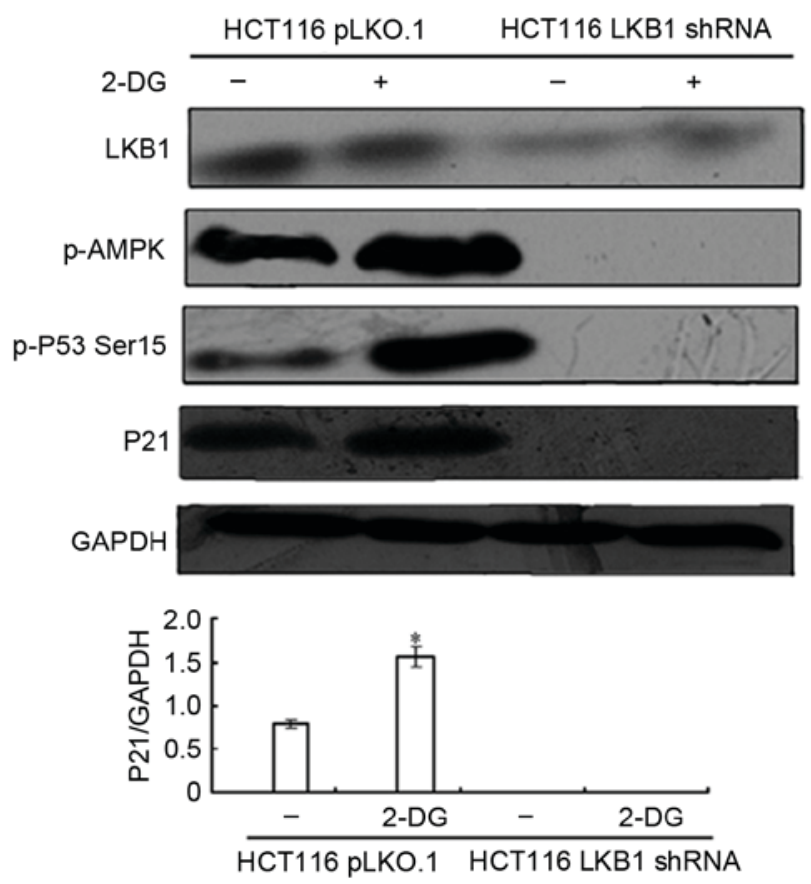

Figure 3. AMPK acts downstream of LKB1 to regulate p21/WAF1 expression level. (A) HCT116 cells were treated with $25 \mathrm{mM}$ 2-DG for $24 \mathrm{~h}$ and western blotting was performed to evaluate p-AMPK Thr172 and p21/WAF1 level, with GAPDH as the loading control. (B) The isogenic HCT116 pLKO.1 and HCT116 LKB1 shRNA cell lines were treated with $25 \mathrm{mM}$ 2-DG for $24 \mathrm{~h}, \mathrm{p}-\mathrm{AMPK}, \mathrm{p}-\mathrm{p} 53 \mathrm{Ser}^{15}$ and p21/WAF1 expression levels were detected by western blotting and GAPDH served as the loading control. ${ }^{*} \mathrm{P}<0.05$ vs. the control. Each blot is representative of three blots obtained from three independent experiments. Results are presented as the mean \pm standard deviation. AMPK, adenosine monophosphate protein kinase; LKB1, liver kinase B1; 2-DG, 2-deoxyglucose; p, phosphorylated; shRNA, short hairpin RNA; WAF1, cyclin dependent kinase inhibitor 1A.

level of p21/WAF1 was analyzed by western blotting. As presented in Fig. 4B, it was revealed that LKB1 depletion by siRNA in p53 wide-type HCT116 cells induced a lower expression level of p21/WAF1. Conversely, in p53-absent HCT116 p53 $^{-/-}$cells, LKB1 transient knockdown did not significantly alter the $\mathrm{p} 21 / \mathrm{WAF} 1$ expression level. Therefore, these results indicate that LKB1-mediated p21/WAF1 induction is p53-dependent.
A

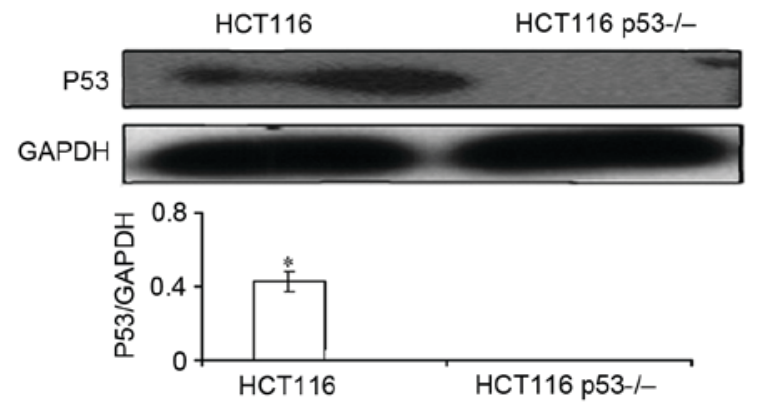

B
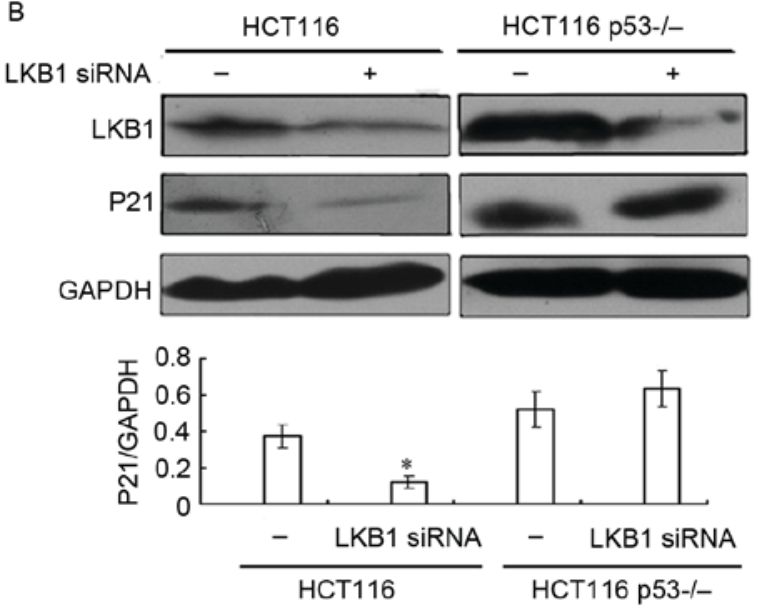

C
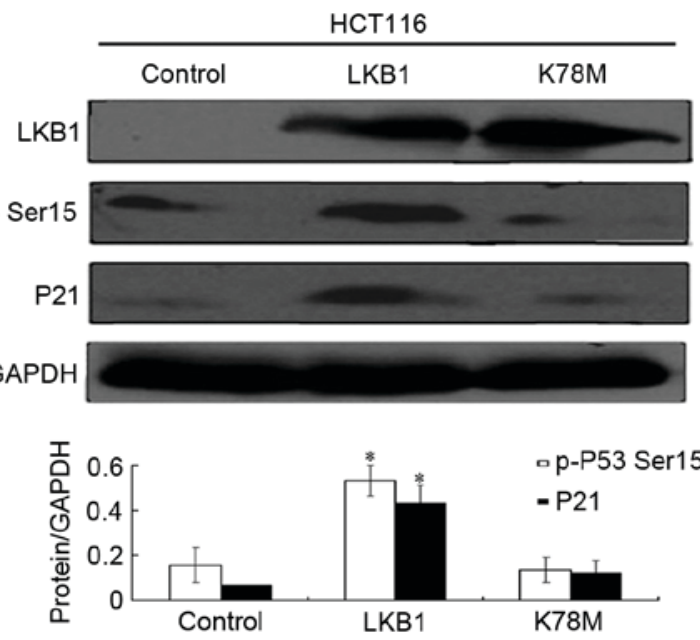

Figure 4. LKB1/AMPK requires p53 to regulate p21/WAF1. (A) P53 protein expression level in P53 wide-type HCT116 colon cell line and the isogenic HCT116 p53 $3^{--}$cells. (B) P53 wild-type HCT116 and the isogenic HCT116 p53 $3^{-1}$ cells were transiently transfected with control siRNA or LKB1 siRNA, and LKB1 and p21/WAF1 expression levels were analyzed using western blotting, with GAPDH used as a loading control. (C) HCT116 cells were transfected with plasmids encoding wild-type LKB1, LKB1 K78M or vector, and western blotting was performed to analyze p-p53 Ser ${ }^{15}$ and p21/WAF1 expression levels. GAPDH serves as the loading control. ${ }^{*} \mathrm{P}<0.05$ vs. the control. Each blot is representative of three blots obtained from three independent experiments. Results are presented as the mean \pm standard deviation. siRNA, short interfering RNA; LKB1, liver kinase B1; AMPK, adenosine monophosphate protein kinase; WAF1, cyclin dependent kinase inhibitor 1A.

To directly evaluate whether LKB1 was involved in p53-Ser ${ }^{15}$ phosphorylation, HCT116 cells were transfected with plasmids encoding wild-type LKB1, LKB1 K78M or 
vector, and western blot analysis was performed to analyze p-p53 Ser ${ }^{15}$ and p21/WAF1 expression levels. As presented in Fig. 4C, wild-type LKB1 significantly increased the expression levels of p-p53 Ser $^{15}$ and p21/WAF1 compared with the control, whereas kinase-deficient LKB1 did not have any significant effects. These results suggest that LKB1 induces the phosphorylation of $\mathrm{p} 53-\mathrm{Ser}^{15}$ in a kinase-dependent manner, which may contribute to the upregulation of p21/WAF1 expression.

\section{Discussion}

Numerous previous studies provide evidence that LKB1 serves an essential role as a tumor suppressor. Tiainen et al (10) demonstrated that LKB1 induced cell cycle arrest and inhibited cell growth by upregulating the p21/WAF1 expression level in LKB1 deficient cervical (HeLa), S3, and melanoma (G361) cancer cell lines. However, it remains unclear whether LKB1 regulates p21/WAF1 expression in other types of cancer cells. The results of the present study confirmed and extended previous observations in lung cancer and colon cancer cells (10-12). Firstly, the present study demonstrated that ectopic LKB1 increased p21/WAF1 expression level in LKB1 mutant NSCLC cells in a kinase-dependent manner. Furthermore, by establishing an isogenic LKB1 stable knockdown colon cell line, it was revealed that LKB1 depletion significantly reduced p21/WAF1 protein expression level. Human LKB1 is a nuclear and cytoplasmic protein. There have been contradicting studies investigating the effect of LKB1 cellular localization on p21/WAF1 expression levels (11-13). Future investigations are required to identify which part of LKB1, localized in the cytoplasm or in the nucleus, serves a more significant role in $\mathrm{p} 21 / \mathrm{WAF} 1$ induction.

AMPK, one of the key substrates of LKB1, is considered as the 'cellular fuel gauge' in sensing and modulating metabolic processes (9). AMPK is a heterotrimeric enzyme composed of two regulatory $\beta$ and $\gamma$ subunits and a catalytic $\alpha$ subunit ( $\alpha 1$ and $\alpha 2$ ) (19). Xu et al (17) demonstrated that AMPK $\alpha 1$ deficiency induced p21/WAF1 reduction in mouse embryonic fibroblasts. Consistent with their findings, the present study revealed that pharmacological activation of AMPK by 2-DG significantly increased the p21/WAF1 expression level, and depletion of LKB1/AMPK impaired the ability of 2-DG to induce p21/WAF1. Thus, the present study identified AMPK as a potential downstream molecule of LKB1 involved in the mediation of $\mathrm{p} 21 / \mathrm{WAF} 1$ induction. We previously revealed that 2-DG treatment potently inhibits the proliferation of LKB1 wild-type lung cancer cells (8). Given the importance of $\mathrm{p} 21 / \mathrm{WAF} 1$ in growth arrest, it has been suggested that 2-DG-mediated p21/WAF1 induction may contribute to the inhibitory effect of the compound. Upregulation of p21/WAF1 by LKB1/AMPK may represent the mechanism underlying growth arrest when cancer cells are exposed to energy stresses with ATP depletion.

Contradicting findings have been reported regarding the role of transcription factor $\mathrm{p} 53$ in $\mathrm{p} 21 / \mathrm{WAF} 1$ induction mediated by LKB1. Tiainen et al (11) and Zeng et al (12) reported that LKB1 required p53 to induce p21/WAF1 expression. In contrast, a previous study by Setogawa et al (20) demonstrated that LKB1 has the potential to induce p21/WAF1 expression in collaboration with LIM domain only 4, GATA binding protein 6 and LIM domain binding, 1 in the p53-deficient HelaS3 cell line (20), suggesting a p53-independent mechanism. The present study confirmed that p53 was required for LKB1-mediated p21/WAF1 induction, as p53 depletion in colon cancer cells were able to inhibit $\mathrm{p} 21 / \mathrm{WAF} 1$ regulation by LKB1. Furthermore, the present study revealed that the phosphorylation of endogenous $\mathrm{p} 53-\mathrm{Ser}^{15}$ was increased by LKB1 overexpression or AMPK activation, suggesting that p53-Ser ${ }^{15}$ phosphorylation, a modification essential for $\mathrm{p} 53$ stabilization, may be involved in p21/WAF1 upregulation. The results of the present study are consistent with previous reports indicating that AMPK induces phosphorylation of p53-Ser15 in hepatoma HepG2 cells (21), mouse embryonic fibroblasts (22), human aortic smooth muscle cells and rabbit aortic strips (23). Other kinases have been demonstrated to phosphorylate p53 at Ser ${ }^{15}$, including ATM and ATR serine/threonine kinases, as they also target p53 at this site (24). Thus, some phosphorylation does occur, despite the fact that the results of the present study demonstrated that LKB1 depletion induced a significant reduction in the expression levels of $\mathrm{p}-\mathrm{p} 53$. Further investigations are required to confirm that LKB1/AMPK-mediated $\mathrm{p} 53-\mathrm{Ser}^{15}$ contributes to p21/WAF1 induction.

In conclusion, the results of the present study demonstrated that in lung and colon cancer cells, LKB1 acts via AMPK to induce p21/WAF1 expression in a p53-dependent manner. Therefore, the present study provides novel molecular insights into the tumor suppressor gene, LKB1.

\section{Acknowledgements}

The authors would like to thank Dr. Wei Zhou (The Winship Cancer Institute, Emory University School of Medicine, Atlanta, GA, USA) for providing the plasmids.

\section{Funding}

The present study was supported by the National Natural Science Foundation of China (grant no. 81572268), Natural Science Foundation of Tianjin (grant no. 17JCYBJC25500), Natural Science Foundation of Tianjin (grant no. 16JCYBJC24400), National Natural Science Foundation of China (grant no. 31301160$)$.

\section{Availability of data and materials}

The datasets used and/or analyzed during the current study are available from the corresponding author on reasonable request.

\section{Authors' contributions}

DZ was responsible for study conception; QM, PX, LS designed and performed the research; QM, PX, LS, and JW analyzed the data; PX, LS and DZ wrote the manuscript.

\section{Ethics approval and consent to participate}

Not applicable. 


\section{Consent for publication}

Not applicable.

\section{Competing interests}

The authors declare that they have no competing interests.

\section{References}

1. Hemminki A, Markie D, Tomlinson I, Avizienyte E, Roth S, Loukola A, Bignell G, Warren W, Aminoff M, Höglund P, et al: A serine/threonine kinase gene defective in Peutz-Jeghers syndrome. Nature 391: 184-187, 1998.

2. Hearle N, Schumacher V, Menko FH, Olschwang S, Boardman LA, Gille JJ, Keller JJ, Westerman AM, Scott RJ, Lim W, et al: Frequency and spectrum of cancers in the PeutzJeghers syndrome. Clin Cancer Res 12: 3209-3215, 2006.

3. Sanchez-Cespedes M, Parrella P, Esteller M, Nomoto S, Trink B, Engles JM, Westra WH, Herman JG and Sidransky D: Inactivation of LKB1/STK11 is a common event in adenocarcinomas of the lung. Cancer Res 62: 3659-3662, 2002.

4. Matsumoto S, Iwakawa R, Takahashi K, Kohno T, Nakanishi Y, Matsuno Y, Suzuki K, Nakamoto M, Shimizu E, Minna JD and Yokota J: Prevalence and specificity of LKB1 genetic alteration in lung cancer. Oncogene 26: 5911-5918, 2007.

5. Zhong D, Guo L, de Aguirre I, Liu X, Lamb N, Sun SY, Gal AA, Vertino PM and Zhou W: LKB1 mutation in large cell carcinoma of the lung. Lung Cancer 53: 285-294, 2006.

6. Gao Y, Ge G and Ji H: LKB1 in lung cancerigenesis: A serine/threonine kinase as tumor suppressor. Protein Cell 2 : 99-107, 2011

7. Alessi DR, Sakamoto K and Bayascas JR: LKB1-dependent signaling pathways. Annu Rev Biochem 75: 137-163, 2006.

8. Dong LX, Sun LL, Zhang X, Pan L, Lian LJ, Chen Z and Zhong DS: Negative regulation of mTOR activity by LKB1-AMPK signaling in non-small cell lung cancer cells. Acta Pharmacol Sin 34: 314-318, 2013.

9. Woods A, Johnstone SR, Dickerson K, Leiper FC, Fryer LG, Neumann D, Schlattner U, Wallimann T, Carlson M and Carling D: LKB1 is the upstream kinase in the AMP-activated protein kinase cascade. Curr Biol 13: 2004-2008, 2003.

10. Tiainen M, Ylikorkala A and Makela TP: Growth suppression by LKB1 is mediated by a G1 cell cycle arrest. Proc Natl Acad Sci USA 96: 9248-9251, 1999.
11. Tiainen M, Vaahtomeri K, Ylikorkala A and Makela TP: Growth arrest by the LKB1 tumor suppressor: Induction of p21(WAF1/CIP1). Hum Mol Genet 11: 1497-1504, 2002.

12. Zeng PY and Berger SL: LKB1 is recruited to the p21/WAF1 promoter by $\mathrm{p} 53$ to mediate transcriptional activation. Cancer Res 66: 10701-10708, 2006.

13. Zhong DS, Sun LL and Dong LX: Molecular mechanisms of LKB1 induced cell cycle arrest. Thorac Cancer 4: 229-233, 2013.

14. el-Deiry WS, Tokino T, Velculescu VE, Levy DB, Parsons R, Trent JM, Lin D, Mercer WE, Kinzler KW and Vogelstein B: WAF1, a potential mediator of p53 tumor suppression. Cell 75: 817-825, 1993.

15. Sun LL, Zhong DS, Wu S, Bai $\mathrm{H}$ and Chen Z: Establishment and gene expression profiling of LKB1 stable knockdown lung cancer cell line. Chin Med J (Engl) 124: 2028-2032,2011.

16. Livak KJ and Schmittgen TD: Analysis of relative gene expression data using real-time quantitative PCR and the 2(-Delta Delta C(T)) method. Methods 25: 402-408, 2001

17. Xu H, Zhou Y, Coughlan KA, Ding Y, Wang S, Wu Y, Song P and Zou MH: AMPK $\alpha 1$ deficiency promotes cellular proliferation and DNA damage via p21/WAF1 reduction in mouse embryonic fibroblasts. Biochim Biophys Acta 1853: 65-73, 2015.

18. Shieh SY, Ikeda M, Taya Y and Prives C: DNA damage-induced phosphorylation of p53 alleviates inhibition by MDM2. Cell 91: 325-334, 1997

19. Hardie DG and Alessi DR: LKB1 and AMPK and the cancer-metabolism link-ten years after. BMC Biol 11: 36, 2013

20. Setogawa T, Shinozaki-Yabana S, Masuda T, Matsuura K and Akiyama T: The tumor suppressor LKB1 induces p21 expression in collaboration with LMO4, GATA-6, and Ldb1. Biochem Biophys Res Commun 343: 1186-1190, 2006

21. Imamura K1, Ogura T, Kishimoto A, Kaminishi M and Esumi H Cell cycle regulation via p53 phosphorylation by a 5'-AMP activated protein kinase activator, 5-aminoimidazole-4-carboxamide-1-beta-D-ribofuranoside, in a human hepatocellular carcinoma cell line. Biochem Biophys Res Commun 287: 562-567, 2001.

22. Jones RG, Plas DR, Kubek S, Buzzai M, Mu J, Xu Y, Birnbaum MJ and Thompson CB: AMP-activated protein kinase induces a p53-dependent metabolic checkpoint. Mol Cell 18: 283-293, 2005.

23. Igata M, Motoshima H, Tsuruzoe K, Kojima K, Matsumura T, Kondo T, Taguchi T, Nakamaru K, Yano M, Kukidome D, et al: Adenosine monophosphate-activated protein kinase suppresses vascular smooth muscle cell proliferation through the inhibition of cell cycle progression. Circ Res 97: 837-844, 2005.

24. Sun B, Ross SM, Rowley S, Adeleye Y and Clewell RA: Contribution of ATM and ATR kinase pathways to p53-mediated response in etoposide and methyl methanesulfonate induced DNA damage. Environ Mol Mutagen 58: 72-83, 2017. 